\title{
OPTIMAL QUADRATURE FORMULAE AND MINIMAL MONOSPLINES IN $L_{q}$
}

\author{
J. KAUTSKY
}

(Received 29 February 1968)

Summary: The quadrature formula of order $m$ using values of derivatives up to the $m-1$ st order with the best possible bound in $L_{a}^{(m)}$ is derived. Using certain properties of the polynomials minimal in $L_{a}$ norm, it is proved that the optimal formula does not use the derivatives of $m-1$ st order if $m$ is even.

\section{Introduction and notation}

Let $L$ be some linear normed space of real functions $f$ on a finite interval $(a, b)$ and let $Q$ be some set of quadrature formulae $Q(f)$ which approximate the integral

$$
I(f)=\int_{a}^{b} f(x) d x
$$

If for any $Q \in \underline{Q}$ the remainder

$$
R_{Q}(f)=I(f)-Q(f)
$$

forms a linear continuous functional on $L$ it is natural to seek that $Q_{0}$ in $\underline{Q}$ for which $\left\|R_{Q_{0}}\right\|_{L^{*}}$ is minimal and to call this $Q_{0}$ the optimal quadrature formula in $\underline{Q}$ with respect to the normed space $L$. While the relation

$$
\left|R_{Q}(f)\right| \leqq\left\|R_{Q}\right\|_{L^{*}}\|f\|_{L}
$$

gives a bound on the error for any quadrature formula, the optimal formula possesses the best possible estimate with respect to the norm in $L$. This norm should be chosen to express our knowledge of the integrated function $t$.

Throughout this paper we shall use the letters $k, m, n$ to denote positive integers;

$$
a, b, c, d, q, q^{\prime}, \quad a_{1}, a_{2}, \cdots, a_{n}, \quad C_{11}, C_{12}, \cdots, C_{k n}
$$

real numbers and we shall assume

$$
\begin{gathered}
-\infty<a<b<\infty \\
-\infty<c<d<\infty \\
1 \leqq q<\infty \\
\frac{1}{q}+\frac{1}{q^{\prime}}=1 \quad \text { if } q>1 \\
a \leqq a_{1}<a_{2}<\cdots<a_{n} \leqq b .
\end{gathered}
$$


Definition 1 . We denote by $\underline{Q}(m, k, n)$ the set of all quadrature formulae of the form

$$
Q(f)=\sum_{i=1}^{k} \sum_{j=1}^{n} C_{i j} f^{(i-1)}\left(a_{j}\right)
$$

such that for $l=0,1, \cdots, m-1$,

$$
R_{Q}\left(x^{l}\right)=I\left(x^{l}\right)-Q\left(x^{l}\right)=0 .
$$

Definition 2. Let $L_{q}^{(m)}$ denote the Banach space of real functions $f(x), x \in(a, b)$, which do not differ by more than a polynomial of degree $m-1$, the $m-1$ 'st derivative of which is absolutely continuous and the $m$ 'th derivative of which is summable with the $q$ th power. The norm in $L_{q}^{(m)}$ is (by definition)

$$
\|f\|_{q, m}=\left(\int_{a}^{b} \cdot\left|f^{(m)}(x)\right|^{q} d x\right)^{1 / q}
$$

Definition 3. We shall say that the knots $a_{j}$ form a semi-equidistant net on $[a, b]$ with ratio $\rho$ if

$$
a_{j}=a+h_{0}+(j-1) h
$$

where

$$
2 h_{0}+(n-1) h=b-a
$$

and

$$
2 h_{0}=\rho h .
$$

The case $\rho=0(\rho=2)$ is called a closed (open respectively) equidistant net.

For $k \leqq m$ and $Q \in Q(m, k, n)$, the remainder $R_{Q}=I-Q$ is obviously algebraically linear on $L_{a}^{(\bar{m})}$. Its continuity is proved in $\S 4$.

It was shown in [1] that the knots of the optimal quadrature formula in $\underline{Q}(2,1, n)$ with respect to the space $L_{q}^{(2)}$ form a semi-equidistant net in $[a, b]$ with the ratio $\rho$ being a function of $q$. The purpose of this paper is to show that the above mentioned optimal quadrature formula is in fact optimal in a larger set $\underline{Q}(2,2, n)$. Furthermore, generalizing this result, we shall derive the optimal quadrature formula in $\underline{Q}(m, m, n)$ with respect to the space $L_{a}^{(m)}$ and prove that this optimal formula is from $\underline{Q}(m, m-1, n)$ for $m$ even.

The last property arises from the relation between the norm and the end-point value of the polynomial of minimal $L_{q}$ norm $(\S 2)$. In $\S 3$ a similar problem is dealt with in terms of minimal monosplines of given discontinuities and the result is applied in $\S 4$ to obtain the required optimal quadrature formulae. 


\section{Polynomials of minimal $L_{q}$-norm}

Among all real polynomials of the form

$$
f(x)=x^{m}+\alpha_{m-1} x^{m-1}+\cdots+\alpha_{0}
$$

there exists a unique one, for which the $L_{a}(a, b)$ norm,

$$
\|f\|_{a}=\left(\int_{a}^{b}|f(x)|^{a} d x\right)^{1 / a}
$$

is minimal. We shall denote this polynomial $p_{m}(x)$, or more precisely $p(m, q, a, b ; x)$. The necessary and sufficient condition for the polynomial (2.1) to be the minimal polynomial $p_{m}(x)$ is (see e.g. [3], 2.8.25, p. 64) to satisfy the so called normal equations

$$
\int_{a}^{b} x^{k}\left|p_{m}(x)\right|^{q-1} \operatorname{sign} p_{m}(x) d x=0, \quad k=0,1, \cdots, m-1 .
$$

Let $N$ denote the norm of the minimal polynomial $p_{m}(x)$ or, more precisely

$$
N=N(m, q, a, b)=\left(\int_{0}^{b}|p(m, q, a, b ; x)| d x\right)^{1 / q} .
$$

The following properties of the minimal polynomial can be easily derived from (2.2):

$$
p_{m}\left(\frac{a+b}{2}+x\right)=(-1)^{m} p_{m}\left(\frac{a+b}{2}-x\right),
$$

i.e. for $m$ even (odd) the minimal polynomial is an even (odd respectively) function with respect to the centre of interval $(a, b)$.

(B) All roots of the minimal polynomial are real, distinct and lie in $(a, b)$.

(C) $p(m, q, c, d ; x)=\left(\frac{d-c}{b-a}\right)^{m} p\left(m, q, a, b ; \frac{(b-a) x+a d-b c}{d-c}\right)$.

(D) $N(m, q, c, d)=\left(\frac{d-c}{b-a}\right)^{(m a+1) / q} N(m, q, a, b)$.

We want to establish the relation between the norm of the minimal polynomial and its value at the endpoint $b$ (or $a$, which is equivalent according to $(A))$. For this purpose let $\beta_{j}, j=1, \cdots, m$ denote the roots of $p_{m}(x)$ in increasing order and let $\beta_{0}=a, \beta_{m+1}=b$. Thus

$$
a=\beta_{0}<\beta_{1}<\cdots<\beta_{m}<\beta_{m+1}=b
$$

and sign $p_{m}(x)=(-1)^{m+j}$ on $\left(\beta_{j}, \beta_{j+1}\right), j=0, \cdots, m$. Integrating by parts we obtain 


$$
\begin{aligned}
N^{a} & =\sum_{j=0}^{m} \int_{\beta_{j}}^{\beta_{j+1}}\left((-1)^{m+j} p_{m}(x)\right)^{q} d x \\
& =\sum_{j=0}^{m}\left\{g\left(\beta_{j+1}\right)-g\left(\beta_{j}\right)-q \int_{\beta_{j}}^{\beta_{j+1}}(-1)^{m+j} p_{m}^{\prime}(x) \cdot x\left|p_{m}(x)\right|^{q-1} d x\right\}
\end{aligned}
$$

where $g(x)=x\left|p_{m}(x)\right|^{q}$.

But $g\left(\beta_{j}\right)=0$ for $j=1, \cdots, m$ and hence

$$
N^{q}=(b-a) p_{m}^{q}(b)-q \int_{a}^{b} x p_{m}^{\prime}(x)\left|p_{m}(x)\right|^{q-1} \operatorname{sign} p_{m}(x) d x .
$$

As $x p_{m}^{\prime}(x)-m p_{m}(x)$ is a polynomial of degree less than $m$, the integral in (2.3) equals $m N^{q}$. We have thus proved the relation

$$
p(m, q, a, b ; b)=\left(\frac{m q+1}{b-a}\right)^{1 / q} N(m, q, a, b) .
$$

Note that the extension of these properties, including $(\mathrm{E})$, for $q$ tending to infinity, i.e. for the uniform norm

is obvious.

$$
\|f\|_{\infty}=\sup _{a \leqq x \leqq b} \operatorname{ess}|f(x)|
$$

\section{Monosplines of minimal $L_{q}$-norm}

Definition 4. Let $m \geqq k$. Any function $g(x)$ defined for all $x$ is said to be an $m$ th degree monospline with discontinuities of order $k$ at $n$ knots on the interval $[a, b]$ if the following conditions hold:

(i) There are $n$ distinct points $a_{j}$ of form (1.1), called knots, such that on each open interval

$$
I_{j}=\left(a_{j}, a_{j+1}\right), j=0, \cdots, n, a_{0}=a_{1}, a_{n+1}=b
$$

$g(x)$ is a polynomial of the form $(2.1)$.

(ii) $g(x) \equiv 0$ outside $[a, b]$.

(iii) If $a<a_{1}, g(x), g^{\prime}(x), \cdots, g^{(m-1)}(x)$ are continuous at $x=a$. Similarly for $b$, if $a_{n}<b$.

(iv) If $k<m, g(x), g^{\prime}(x), \cdots, g^{(m-k-1)}(x)$ are continuous at $x=a_{1}, a_{2}, \cdots, a_{n}$.

Thus a. monospline is piece-wise an $m$ 'th degree polynomial on $[a, b]$ and vanishes elsewhere. The case $k=1$ corresponds to the usual meaning given to the word 'monospline' (compare, e.g. [2]), i.e. only the $m-1$ 'st derivative is allowed to have discontinuities at the knots. When $k=2$, both $m-1$ 'st and $m-2$ 'nd derivatives of $g(x)$ may have discontinuities at knots, etc.; for $k=m$ the local polynomials defining $g(x)$ on $I_{j}$ 
$(j=2, \cdots, m-1)$ are independent and we consider two monosplines being equal if they differ only at knots.

Lemma. Let $A, B, C$ be positive real numbers and $\alpha>1$. The function

$$
F\left(x_{0}, x_{1}, \cdots, x_{n}\right)=A\left(x_{0}^{\alpha}+x_{n}^{\alpha}\right)+B \sum_{j=1}^{n-1} x_{j}^{\alpha}
$$

with the domain of definition given by

$$
\sum_{j=0}^{n} x_{j}=C, \quad x_{j} \geqq 0, \quad j=0, \cdots, n
$$

reaches its minimal value

$$
F_{\min }=B \cdot C^{\alpha}(\rho+n-1)^{1-\alpha}
$$

uniquely at the point

where

$$
2 x_{0}=2 x_{n}=\rho x_{j}=C \rho /(\rho+n-1), \quad j=1,2, \cdots, n-1,
$$

$$
\rho=2(B / A)^{1 /(\alpha-1)} .
$$

Proof is by elementary calculus.

THEOREM 1. Among all $m$ 'th degree monosplines with discontinuities of order $m$ at $n$ knots on the interval $[a, b]$ there is exactly one that has minimal $L_{a}$ norm. The knots of this minimal spline form a semi-equidistant net with the ratio $\rho$ (independent of $n$ ) given by (3.2); the minimal norm is given by (3.1). Further, if $m$ is even, the minimal monospline has discontinuities of the order $m-1$ only.

Proof. By definition 4 an arbitrary spline $g(x)$ having knots (1.1) must be of the form

$$
\begin{aligned}
& g(x)=(x-a)^{m} \text { on } I_{0}, \\
& g(x)=(x-b)^{m} \text { on } I_{n} \text { and } \\
& g(x) \text { of the type }(2.1) \text { on } I_{j}, \quad j=1, \cdots, n-1 .
\end{aligned}
$$

The $q^{\prime}$ th power of its $L_{a}$ norm, which we may minimize instead of the norm itself, is

$$
N^{a}(g)=\int_{a}^{b}|g(x)|^{a} d x=\sum_{j=0}^{n} \int_{I_{s}}|g(x)|^{a} d x
$$

As the local polynomials defining $g(x)$ on the intervals $I_{j}$ are independent, we can divide the minimization of $N^{a}(g)$ into two steps: firstly, we find the minimal monospline, say $g_{0}$ for each fixed net. Secondly we minimize $N^{q}\left(g_{0}\right)=F\left(h_{0}, h_{1}, \cdots, h_{n}\right)$ as a function of the lengths $h_{j}=a_{j+1}-a_{j}$ of intervals $I_{j}, j=0,1, \cdots, n$, in the domain given by 


$$
\sum_{j=0}^{n} h_{j}=b-a, \quad h_{0} \geqq 0, h_{n} \geqq 0
$$

and

$$
h_{j}>0, \quad j=1, \cdots, n-1 .
$$

However, to establish the first step, we have only to set

$$
g_{0}(x)=p\left(m, q, a_{j}, a_{j+1} ; x\right) \text { on } I_{j}, \quad j=1, \cdots, n-1 .
$$

Using property (D) of the minimal polynomials and denoting

we obtain

$$
N(m, q)=N(m, q,-1,1)
$$

$$
\begin{aligned}
F\left(h_{0}, \cdots, h_{n}\right)= & N^{q}\left(g_{0}\right)=\int_{a}^{a_{1}}\left|(x-a)^{m}\right|^{q} d x+\int_{a_{n}}^{b}\left|(x-b)^{m}\right|^{q} d x \\
& +\sum_{j=1}^{n-1} N^{q}\left(m, q, a_{j}, a_{j+1}\right) \\
= & \frac{1}{m q+1}\left(h_{0}^{m a+1}+h_{n}^{m q+1}\right)+N^{q}(m, q) \sum_{j=1}^{n-1}\left(\frac{h_{j}}{2}\right)^{m q+1}
\end{aligned}
$$

For the second step we use the above mentioned lemma to find the norm $N_{\min }$ of the minimal monospline and the ratio $\rho$ of its semi-equidistant net of knots:

$$
\begin{aligned}
N_{\text {min }} & =\left(\frac{b-a}{2}\right)^{m+(1 / a)} \frac{N(m, q)}{(\rho+n-1)^{m}} \\
\rho & =N^{1 / m}(m, q)\left(\frac{m q+1}{2}\right)^{1 / m q}
\end{aligned}
$$

To complete the proof of Theorem 1 we have to show that for $m$ even the minimal monospline, say $g_{1}(x)$, is continuous at the knots. Denoting $p(m, q ; x)=p(m, q,-1,1 ; x)$ and using property $(\mathrm{C})$ we have

$$
g_{1}(x)=\left(\frac{h}{2}\right)^{m} p\left(m, q ; \frac{2 x-a_{j}-a_{j+1}}{h}\right) \text { on } I_{j}, \quad j=1, \cdots, n-1,
$$

where

$$
h=\frac{b-a}{\rho+n-1}
$$

is the step of the semi-equidistant net. As $p(m, q ; x)$ is an even function for $m$ even, the continuity of $g_{1}(x)$ at $a_{j}$ is obvious for $j=2,3, \cdots, n-1$. The continuity at $a_{1}$ (and at $a_{n}$ ) follows from the property (E): 


$$
\begin{aligned}
g_{1}\left(a_{1}+\right) & =\left(\frac{h}{2}\right)^{m} p(m, q ;-1) \\
& =\left.\left(\frac{h_{0}}{\rho}\right)^{m}\left(\frac{m q+1}{2}\right)^{1 / q}\right|_{N}(m, q)=h_{0}^{m} \\
& =g_{1}\left(a_{1}-\right) .
\end{aligned}
$$

\section{Optimal quadrature formulae in $L_{q}^{(m)}$}

THEOREM 2. Let $k \leqq m$. There is a one-to-one corresponaience between the set of quaarature formulae $\underline{Q}(m, \dot{k}, n)$ and the set of all $m$ 'th deyree monosplines with discontinuities of order $k$ at $n$ knots in the inierval $[a, b]$, which satisfies the following conaitions:

(i) The knots $a_{j}, j=1, \cdots, n$ are common both to $\underline{Q} \in Q(m, k, n)$ and to the corresponding monospline $g(x)$.

(ii) If $f=L_{q}^{(m)}$ and $C_{i j}$ are coefficients of $Q$, then

$$
\begin{aligned}
R_{Q}(f) & =\int_{a}^{b} f(x) d x-\sum_{i=1}^{k} \sum_{j=1}^{n} C_{i j} f^{(i-1)}\left(a_{j}\right) \\
& =\frac{(-1)^{m}}{m !} \int_{a}^{b} f^{(m)}(x) g(x) d x .
\end{aligned}
$$

(iii)

$$
C_{i j}=\frac{(-1)^{i}}{m !}\left(g^{(m-\imath)}\left(a_{j}+\right)-g^{(m-i)}\left(a_{j}-\right)\right), \quad j=1, \cdots, n, i=1, \cdots, k .
$$

Proof of Theorem 2 will only be outlined here for the theorem is only a modification of known properties of the remainders of quadrature formulae. Given a monospline $g(x)$ we may integrate by parts the integral

$$
\int_{a}^{b} f^{(m)}(x) g(x) d x
$$

We find that the quadrature formula $Q$ with the same knots as $g(x)$ and coefficients $C_{i j}$ given by (4.2) satisfies (4.1). Therefore its order of precision is $m$ and because of the order of discontinuity of $g(x), C_{i j}=0$ for $i>k$. Thus $Q \in \underline{Q}(m, k, n)$. On the other hand, given $Q \in \underline{Q}(m, k, n)$, we will construct a function $g(x)$ in the following way:

$$
g(x) \equiv 0 \text { on }(-\infty, a), g(x)=(x-a)_{m} \text { on } I_{0}, g(x) \text { of type }(2.1) \text { on } I_{1}
$$

to satisfy $(4.2)$ for $j=1$ (with $C_{i j}=0$ for $i>k$ ), and similarly for $I_{j}$, $j=2, \cdots, n$; finally, we set $g(x) \equiv 0$ on $(b, \infty)$. This function $g(x)$ satisfies all conditions of a monospline. The restrictions required at point $b$ are easily proved from the fact that $Q$ has the order of precision $m$. 
Corollary. Since $g \in L_{a^{\prime}}$ and (4.1) holds, $R_{Q}(f)$ forms a continuous linear functional on $L_{q}^{(m)}$ for any $Q \in \underline{Q}(m, k, n)$.

COROLLARY. The knots of the quadrature formula in $\underline{Q}(m, m, n)$ which is optimal with respect to the space $L_{q}^{(m)}$ form a semi-equidistant net

$$
a_{j}=a+(j-1+\rho / 2) h
$$

with ratio $\rho$ given by (3.2) and step $h$ by (3.4). The coefficients of the optimal formula are ((3.3) and (4.2))

and

$$
C_{i \mathbf{1}}=(-1)^{i+1} C_{i n}=\left(\frac{h}{2}\right)^{i}\left(\frac{1}{m !} p^{(m-i)}(m, q ; 1)-\frac{(-1)^{i}}{i !} \rho^{i}\right)
$$

$$
C_{i 2}=C_{i 3}=\cdots=C_{i n-1}=C_{i}=\frac{1-(-1)^{i}}{m !}\left(\frac{h}{2}\right)^{i} p^{(m-i)}(m, q ; 1) .
$$

If $m$ is even, $C_{m j}=0$ for $j=1, \cdots, n$ and thus $Q_{\mathrm{opt}} \in \underline{Q}(m, m-1, n)$. The error estimate of the optimal quadrature formula is

$$
\left|R_{Q_{\mathrm{opt}}}(f)\right| \leqq N_{\min }|| f \|_{\boldsymbol{Q}^{\prime}, m},
$$

where $N_{\min }$ is from (3.1).

We can give the explicit forms of the derivatives of minimal polynomials $p(m, q ; x)$ for some values of $q$. In particular if $q=2, \infty, 1$, $p(m, q ; x)$ are the Legendre, Chebyshev first and Chebyshev second order polynomials respectively. Then

$$
\begin{aligned}
& p^{(l)}(m, 2 ; 1)=\left(\begin{array}{l}
m+l \\
m-l
\end{array}\right) \frac{\left(\begin{array}{c}
l-\frac{1}{2} \\
l
\end{array}\right)}{\left(\begin{array}{c}
m-\frac{1}{2} \\
m
\end{array}\right)} l ! 2^{l-m} \\
& p^{(l)}(m, \infty ; 1)= \begin{cases}m\left(\begin{array}{c}
m+l-1 \\
m-l
\end{array}\right)(l-1) ! 2^{l-m} & \text { for } l>0 \\
2^{1-m} & \text { for } l=0\end{cases} \\
& p^{(l)}(m, 1 ; 1)=\left(\begin{array}{c}
m+l+1 \\
m-l
\end{array}\right) l ! 2^{l-m}
\end{aligned}
$$

and the knots and coefficients of corresponding optimal quadrature formulae can be easily calculated. Even though these three minimal polynomials are special cases of Jacobi polynomials, the author's opinion, based on results of numerical computation, is that in general the minimal polynomials $p(m, q ; x)$ are not Jacobi polynomials. 


\section{References}

[1] V. I. Krylov, Approximate Calculations of Integrals (MacMillan, New York, 1962, Translated from Russian).

[2] I. J. Schoenberg, 'On Interpolation by Spline Functions and its Minimal Properties', On Approximation Theory, Proc. of the Conference, August 4-10, 1963, ISNM, Vol. 5.

[3] A. F. Timan, Theory of Approximations of Functions of a Real Variable, (Pergamon Press, 1963, Translated from Russian).

Cf Discipline of Applied Mathematics

The Flinders University of South Australia 